OPEN ACCESS

Edited by:

Valentin Sebastian Schäfer,

University Hospital Bonn, Germany

Reviewed by:

Anabela Barcelos,

New University of Lisbon, Portugal

Cheng-De Yang,

Shanghai Jiao Tong University, China

*Correspondence:

Michael Schirmer

michael.schirmer@i-med.ac.at

TORCID:

Johannes Pallua

orcid.org/0000-0003-0203-213X

Michael Schirmer

orcid.org/0000-0001-9208-7809

Specialty section:

This article was submitted to

Rheumatology,

a section of the journal

Frontiers in Medicine

Received: 11 August 2021

Accepted: 28 September 2021

Published: 25 October 2021

Citation:

Pallua J and Schirmer M (2021) Identification of Five Quality Needs for

Rheumatology (Text Analysis and

Literature Review).

Front. Med. 8:757102.

doi: 10.3389/fmed.2021.757102

\section{Identification of Five Quality Needs for Rheumatology (Text Analysis and Literature Review)}

\author{
Johannes Pallua ${ }^{1,2+}$ and Michael Schirmer ${ }^{3 * t}$ \\ ${ }^{1}$ University Hospital for Orthopedics and Traumatology, Medical University of Innsbruck, Innsbruck, Austria, ${ }^{2}$ Fachhochschule \\ Gesundheit, Health University of Applied Sciences Tyrol, Innsbruck, Austria, ${ }^{3}$ Department of Internal Medicine, University \\ Clinic II, Innsbruck Medical University, Innsbruck, Austria
}

Background: While the use of the term "quality" in industry relates to the basic idea of making processes measurable and standardizing processes, medicine focuses on achieving health goals that go far beyond the mere implementation of diagnostic and therapeutic processes. However, the quality management systems used are often simple, self-created concepts that concentrate on administrative processes without considering the quality of the results, which is essential for the patient. For several rheumatic diseases, both outcome and treatment goals have been defined. This work summarizes current mainstreams of strategies with published quality efforts in rheumatology.

Methods: PubMed, Cochrane Library, and Web of Science were used to search for studies, and additional manual searches were carried out. Screening and content evaluation were carried out using the PRISMA-P 2015 checklist. After duplicate search in the Endnote reference management software (version X9.1), the software Rayyan QCRI (https://rayyan.qcri.org) was applied to check for pre-defined inclusion and exclusion criteria. Abstracts and full texts were screened and rated using Voyant Tools (https:// voyant-tools.org/). Key issues were identified using the collocate analysis.

Results: The number of selected publications was small but specific (14 relevant correlations with coefficients $>0.8$ ). Using trend analysis, 15 publications with relative frequency of keywords $>0.0125$ were used for content analysis, revealing 5 quality needs. The treat to target (T2T) initiative was identified as fundamental paradigm. Outcome parameters required for T2T also allow quality assessments in routine clinical work. Quality care by multidisciplinary teams also focusing on polypharmacy and other quality aspects become essential, A global software platform to assess quality aspects is missing. Such an approach requires reporting of multiple outcome parameters according to evidence-based clinical guidelines and recommendations for the different rheumatic diseases. All health aspects defined by the WHO (physical, mental, and social health) have to be integrated into the management of rheumatic patients. 
Conclusion: For the future, quality projects need goals defined by T2T based initiatives in routine clinical work, secondary quality goals include multidisciplinary cooperation and reduction of polypharmacy. Quality indicators and standards in different health systems will provide new information to optimize patients' care in different health systems.

Keywords: quality management (QM), medical care, quality dimensions, health, musculoskeletal diseases, rheumatology, immunology

\section{INTRODUCTION}

Widespread attention to the quality of universal health care began in 2001 with a report by the Institute of Medicine (IOM) (1). This report identified the general public's interest, clinicians, and decision-makers, emphasizing morbidity and mortality due to medical errors. This report highlighted the need to restructure the health system to provide better quality and safer care. Accordingly, more and more attention was drawn to improve quality and safety in rheumatological health care in the past two decades. Rheumatology includes about 200 different, often rare diseases. In addition to this list of different diagnoses, the chronicity of most of these rheumatological diseases is also a particular challenge for care management.

Rheumatologists mainly focus on specific quality issues related to diagnosis and the effectiveness of therapeutic interventions. Adherence to national and international guidelines certainly increases the quality aspect of effectiveness and improve safety issues (2). Additional quality goals include patient safety, timely and patient-centered care, as well as effective, efficient and socially fair health care (1,3-11). An expanded view of quality issues may also contribute to more attractive health care providers' work areas.

Many health organizations have developed quality indicators (QIs) to monitor, measure, and manage their health systems' performance and ensure effectiveness, efficiency, safety, timeliness, patent satisfaction, and equal access (12-14). Besides, a QI has to meet clinical relevance, scientific acceptance, and applicability (15) and be reliable and validated (16). Thus, QIs can independently reflect the actual performance of health organizations (17).

According to Donabedian's conceptual model, key QIs can be classified by referring to the three components of the health system's structure, process, and outcome. While the structure describes the context in which health care is provided (e.g., hospital buildings, staff, funding, and equipment), the processes encompass all transactions between patients and service providers throughout health care, and the results relate to the effects of health care on patients' health (18). All three components of this conceptual model should also be realized in rheumatology. In rheumatology, QIs for measuring the desired therapeutic achievements were used for both adults $(19,20)$ and pediatric rheumatology $(21,22)$. However, QIs and performance reports are descriptive and do not directly lead to any improvements in performance. Therefore, strategies are necessary for clinical routine, understanding the fundamentals of quality problems and practical tools to assure ongoing improvements of clinical outcomes. Accordingly, there is increasing interest in using electronic health records (EHRs) to develop and use electronic clinical quality measures (eCQMs) in rheumatology $(23,24)$. eCQMs are a new automated approach to extract information on QIs from the EHRs (23). Thus, in combination with data analyses from national clinical data registries, eCQMs not necessarily reduce the burden of collecting appropriate data, but they can serve as valuable tools for continuous quality improvement. Critical technological advances in building the infrastructure for the implementation of eCQMs will advance this field also in rheumatology (25).

It can be anticipated that outcome-oriented clinical quality management will become a standard procedure in the future and represent an essential pre-requisite to objectify medical competition-also in rheumatology. This work identifies and summarizes EHR-based quality needs for future efforts in rheumatology.

\section{METHODS}

A literature search was performed in the PubMed and Cochrane libraries as well as in the Web of Science search platforms in February 2021. The search was carried out using the following terms: "(rheumatology OR musculoskeletal diseases OR vasculitis OR arthritis OR connective tissue diseases) AND (quality of health care OR quality assurance health care) AND electronic health records." In addition, a manual search was performed to identify additional potentially relevant articles.

English literature was included if published between 2000 and 2020. Non-peer-reviewed articles, case reports, comments, and conference summaries were excluded. A duplicate search was performed in the Endnote software (version X9.1) and the Rayyan QCRI software (https://rayyan.qcri.org) (26), together with manual screening of titles and abstracts based on specified inclusion and exclusion criteria. The literature screening and evaluation were carried out according to the PRISMA-P 2015 checklist (27).

The text mining software Voyant Tools (https://voyant-tools. org/) was used to read and analyze titles and abstracts. Analyses include a comparison of several documents, providing results from the Cirrus, the Collocates Graph, the Correlation, the Mandala and the Scatter Plot Tool together with a Trend analysis as follows (28):

- The Cyrrus Tool visualizes frequently used words of the documents as a word cloud, with words sized and positioned 
according to the frequency of their use. Color and absolute position of words are not significant.

- The Correlation Tool calculates the Pearson correlation coefficients by comparing the relative frequencies of terms (relative to each document for the corpus) (29). A coefficient that approaches 1 indicates that values correlate positively; approaching -1 indicates that values correlate negatively.

- The Collocates Graph represents keywords and terms that occur in close proximity as a force-directed network graph.

- The Trend analysis represents frequencies of terms across documents. Each series in the graph is colored according to the word it represents. At the top of the graph, a legend displays which words are associated with which colors. A cutoff of 0.0125 was chosen as relevant relative frequency for key terms, to be included into the qualitative analysis of the main quality issues.

The qualitative content analysis of the key quality issues for rheumatology was based on the trend analysis results.

\section{RESULTS}

A total of 1,251 records were included in the title/abstract screening of Rayyan QCRI (https://rayyan.qcri.org), including 21 records identified by manual research and after removal of 10 duplicates. Most of the articles came from the United States ( $n$ $=241)$ followed by the UK $(n=124)$, Canada $(n=69)$, and Australia $(n=61)$.

Figure 1 demonstrates the flow diagram for the PRISMA-P strategy. One thousand sixty-nine records were excluded because they were irrelevant to the pre-defined questions, and 182 remaining full texts were checked for suitability using the PRISMA-P 2015 checklist.

\section{Full Text Analysis}

Forty-three full texts (17 articles and 26 reviews) were included in the text analysis using the text mining software Voyant (https:// voyant-tools.org/).

\section{Cyrrus Analysis}

The most frequently used terms in the selected publications are visualized in Figure 2. Frequencies ranged from 142 to 33 per text file. This analysis shows that the literature search was carried out specifically for patients' care and quality (with 142, 89, and 108 nominations for patients, care and quality, respectively).

\section{Correlation Analysis}

Table 1 shows the Pearson correlation coefficients between the single-word citations in the selected publications. According to the search strategy, the strongest correlations exist between words related to the concept of "quality."

\section{Collocate Graph}

The Collocate Graph represents keywords and terms that occur near a force-directed network graph. The relationship between various terms is shown as a network according to the selected literature in Figure 3.
This network analysis shows that according to quality-related literature the terms "physical," "psychological," and "social" (as used by the WHO to define the status of "health"), together with the term of "education" are separated from the remaining terms. Independent from this finding, a strong linkage exists not only between the terms "EHR," "quality," and "outcome," but also with the Donabedian dimensions of "structure" and "process." Even the terms "management" and "care" are rarely connected with "treat to target" so far. Based on this analysis, it can be assumed that there is a strong correlation between the quality dimensions in the selected articles, but links of these dimensions with the WHO-defined health goals and education could not be identified in the literature.

\section{Trend Analysis}

The results of the trend analysis are shown in Figure 4. A total of 15 publications ( 11 articles and 4 reviews) showed a relative frequency of key terms which was higher than 0.0125 in the trend analysis.

Out of these publications, 1 article on structure (31), 7 articles on process $(24,32-37)$ and 2 articles on outcome quality $(31,38)$ were identified. For the WHO-defined health goals, 1 article on physical (39), no article on psychological and 1 article on social issues (40) were identified. One for the role of nursing (41), for polypharmacy 1 (42), and for treat to target (43) 1 publication was found in each case.

\section{Key Issue Analysis}

The trend analysis for the content-related aspects identified five key issues for further content analysis. These key issues are outlined in the following paragraphs:

\section{Quality Indicators for Clinical Routine}

Measurements of outcome using validated outcome parameters are necessary to assess and further improve health care quality and have gained increasing momentum since gaps in the quality of care became evident in the USA and other Western countries (44). During the last decades, various tools were developed to improve health care quality like clinical practice guidelines, QIs and quality standards (QS).

A clinical practice guideline is usually defined as a systematically developed statement to assist physicians and patients in decision-making in specific circumstances according to best available evidence and/or expert consensus $(45,46)$. While clinical practice guidelines aim to improve consistency of care and compliance with evidence-based practice (46), compliance with clinical guidelines is not easy to measure (47).

QIs are specific statements that are often made in the following standard format: IF a specific clinical scenario occurs, THEN a specific action should be taken (48). A simplified example of a QI commonly used in rheumatology reads: "If a patient has RA, a disease-modifying anti-inflammatory drug should be prescribed" (2017). For other chronic conditions such as diabetes mellitus and high blood pressure, QIs are easy to use to quantify the quality of care. For these chronic diseases, the availability of a single measure as a gold standard makes it easier to measure the quality of care. However, in most 


\section{Identification of studies via databases and registers}

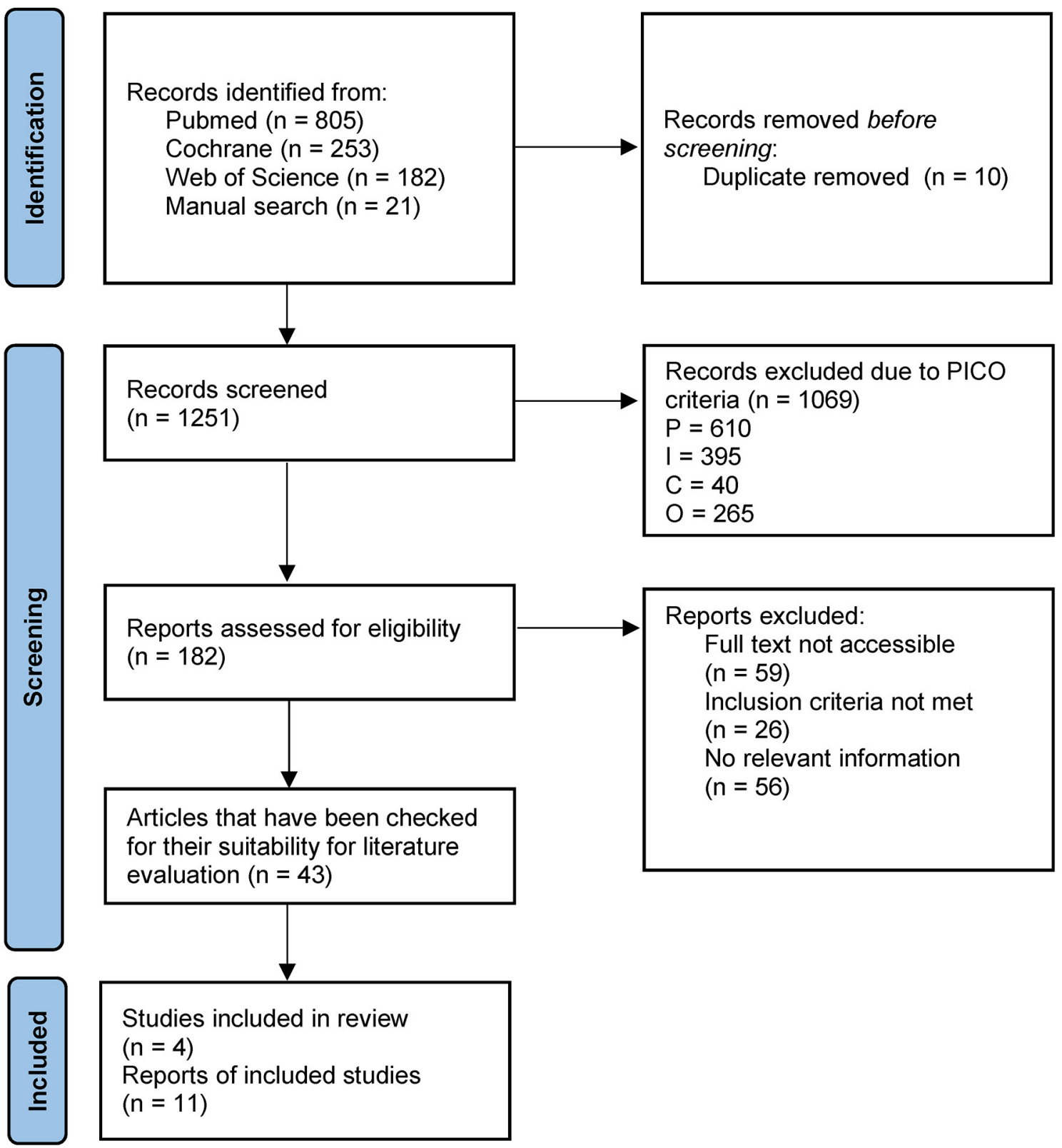

FIGURE 1 | Flow diagram of records' selection according to the PRISMA-P strategy (27, 30).

rheumatic diseases, objective clinical, laboratory or imaging parameters alone cannot be used as gold standards $(49,50)$. Therefore, large datasets are required to identify an initial set of QIs separately for each rheumatic disease. A fascinating study was carried out with a huge sample of RA-patients from clinical practices in nine countries (51). The primary purpose of the database was to support clinical management and not to assess compliance with pre-defined QIs. The number of documented examinations differed significantly from country to country, which limited the comparison of compliance with QIs between countries. The main limitation of this study was that the database used was developed in 2007 before the QIs were created. The transfer of QIs between countries is difficult because of the geographical differences in clinical practice (31, 52). Another study addressed the implementation of structural, processual, and outcome-aspects of QIs to monitor rheumatoid arthritis (RA) (31). As this work was based on internationally published studies and guidelines, these authors suggested that 


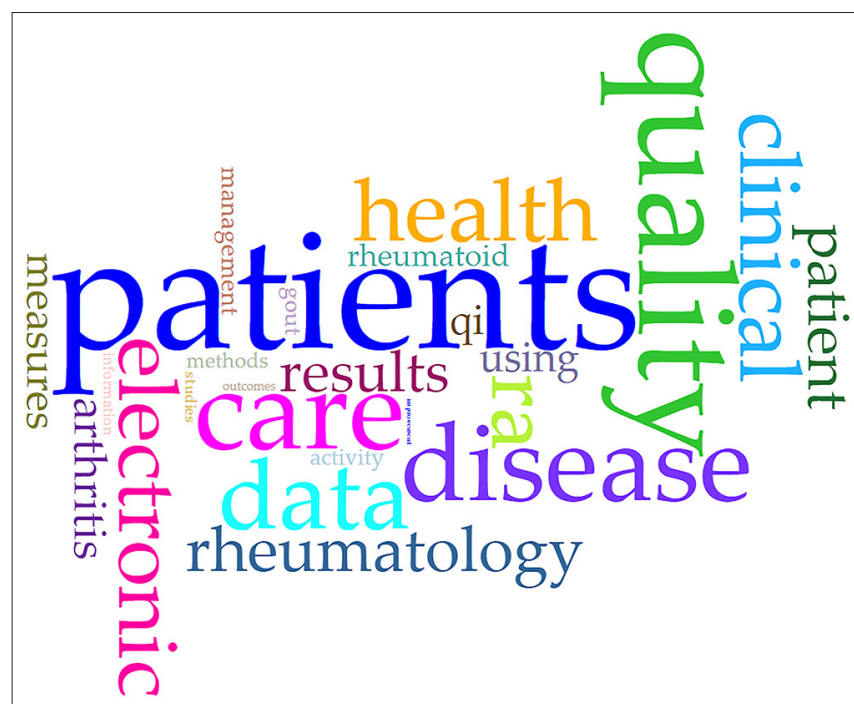

FIGURE 2 | Cyrrus plot showing most frequently used terms of the literature search.

TABLE 1 | Pearsons correlation coefficients between single words of the literature search.

\begin{tabular}{llc}
\hline Term 1 & Term 2 & Correlation \\
\hline Benchmarking & Treatment & 1.0 \\
Electronic & Quality & 1.0 \\
Indicator & Quality & 1.0 \\
Medical doctor & Quality & 1.0 \\
Arthritis & Electronic & 0.9370426 \\
Patient & RA & 0.9370426 \\
Improvement & Quality & 0.8989332 \\
Arthritis & Quality & 0.8291562 \\
Benchmarking & Quality & 0.8291562 \\
Treatment & Quality & 0.8291562 \\
Improvement & Quality & 0.8291562 \\
Indicator & Quality & 0.8291562 \\
Examination & Quality & 0.80403024 \\
Data & Quality & 0.80178374 \\
\hline Acort & &
\end{tabular}

A correlation coefficient that approaches 1 indicates that values correlate positively. $R A$, rheumatoid arthritis.

only the "structural" QIs could vary worldwide due to different health systems.

In a comprehensive review of QS for inflammatory arthritis, significant heterogeneity was described for the methodology used to develop a QS (47). The methods ranged from expert discussions to formally modified Delphi methods, QSs were often proposed but not well-defined, and patients were not always involved in developing these QSs. Consequently, the authors recommended clear criteria for the development of QSs, as the introduction of all available standards is unlikely to be useful or necessary. In addition, the development of QSs should go along with the proposal of QIs to measure quality improvements. For adult spondyloarthritis (SpA), the international ASAS group of experts has developed a QS to improve health care services (37). These standards provide a clear description of the high-priority areas for quality improvement and monitoring. As part of this work, QS was formulated for the critical areas of referral, rheumatology assessment, treatment, education/self-management, and comorbidities. One of the authors' considerations was the transfer of this QS to other rheumatological diseases. However, as this QS refers to SpA and may not represent other rheumatological diseases, it is suggested that this QS is not directly transferred to other rheumatic diseases and can only serve as a template for other diseases. Unfortunately, the feasibility of this QS has not yet been tested in clinical practice anywhere in the world.

\section{Treat to target (T2T) as a treatment strategy}

The treatment target of clinical remission or at least a state of low disease activity was initially introduced into rheumatology for the treatment of RA, requiring frequent reassessment of disease activity and drug adjustments (53). Later on, the principles of T2T were also used for other guidelines (54-56). For the implementation process of $\mathrm{T} 2 \mathrm{~T}$ into clinical routine, audits and feedback loops are required $(57,58)$.

Implementation of the T2 $\mathrm{T}$ strategy takes time, effort, and a good health system infrastructure, including platforms of EHRs, to support tracking of patients with follow-ups after specific therapeutic interventions. With subsequent audits and feedback, quality reports to rheumatologists can then initiate improved routines in rheumatology clinics.

Indeed, one initiative to optimize T2T strategies using audit and group feedback for RA provided deep insights into the implementation process: T2T resulted in a high measurement rate of disease activity recorded by an electronic platform, with suboptimal follow-up times identified for patients without remission, especially for patients with long disease duration (36). Three areas for potential quality improvement were identified during the group feedback session: (1) expanding the use of an electronic data collection software, (2) the need for a protocol to manage early RA, and (3) expanding similar initiatives to patients with other diagnoses. Limitations of this study were missing completeness of all outcome measurements, lack of relating outcome measurements to time from diagnosis to start of therapy (although the delay in treatment can affect the likelihood of remission or a state of low disease activity), and the singlecenter design (despite an excellent electronic infrastructure). For improved documentation of the actual medication consumption, the authors proposed a link to medication dispensing by pharmacies. Also, socio-demographic factors with the insurance status of the patients should be available.

Another study on the implementation of T2T examined the effect of collaborative training to facilitate the implementation of T2T, using the quality circles of plan-do-check-act (PDCA) (43). Designed as a continuous improvement model, phase 1 was a randomized clinical control study in which the intervention was compared with a control group, whereas in phase 2 the control group phased the jointly developed training intervention 


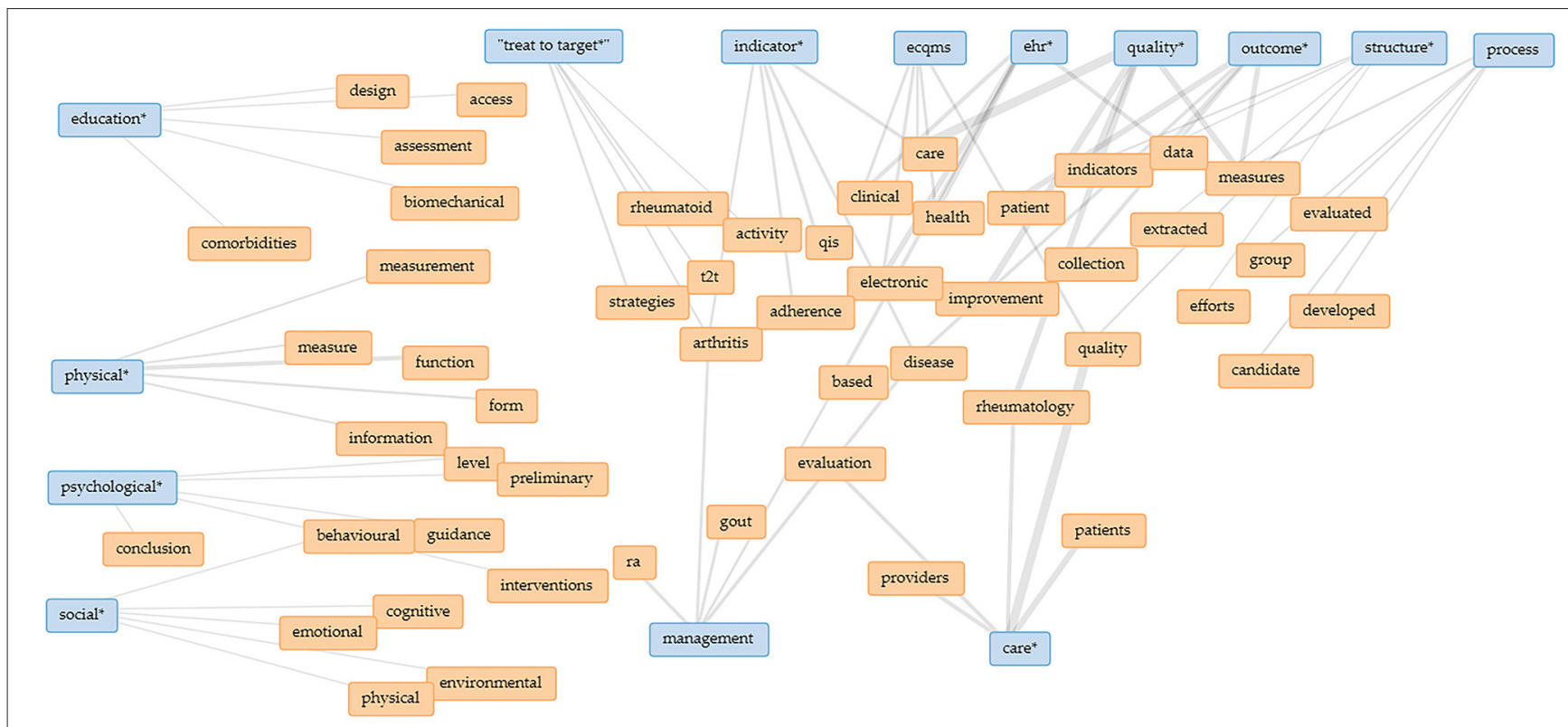

FIGURE 3 | Network of terms identified by the Collocate Graph Tool. The group of "education," "physical," "psychological," and "social" is clearly separated from the group of "management" and "care" as well as the group of "treat to target," "indicator," "ecqms," "EHR," "quality," "outcome," "structure," and "process." ecqm, electronic clinical quality measures; ehr, electronic health record; ra, rheumatoid arthritis; qis, quality indicators; t2t, treat to target. "including similar items.

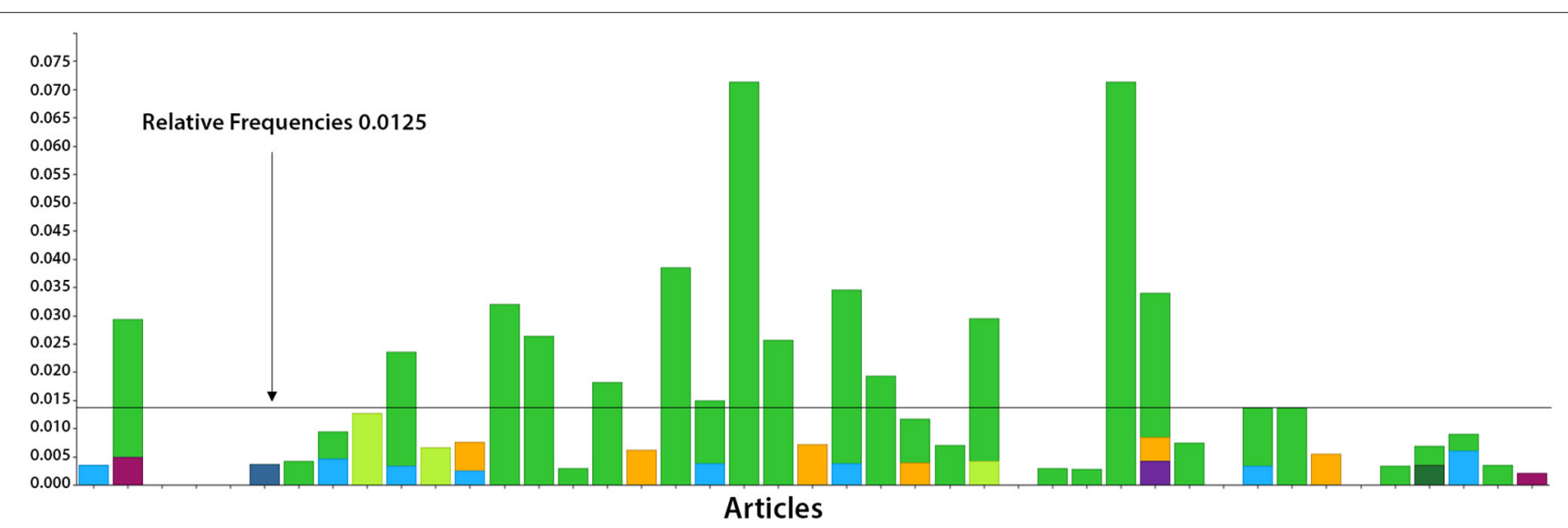

FIGURE 4 | Trend analysis of the 43 full texts included related to quality (in green color), the Donabedian quality dimensions ("structure," "process," and "outcome," shown in violet, light blue and orange color, respectively) and the WHO-defined health goals ("physical," "mental," and "social," shown in light green, dark blue and dark green color, respectively). The cut-off of 0.0125 is shown as a line, indicating a relevant relative frequency.

of phase 1. In contrast, the interventional group of phase 1 was further observed. During the PDCA cycles, T2T implementation was checked, but specific individual patient care problems were not observed. The strength of this joint training intervention was the collaboration between 11 different locations, facilitating the improvement and documentation of care, disease activity assessments, and treatment decision-making. Limitations were the high expenditure of time, checking the T2T goals being met, difficulties of generalizing documentation on the platform for EHRs and the location-specific nature of team meetings. This study confirmed that optimal RA management requires continuous monitoring of disease activity and continuous adjustment of treatment to maintain disease control.

There are several limitations to the T2T strategy: First, T2T requires process improvement with regular measurement of disease activity and timely adjustment of treatments, which is difficult without adequate support by cooperative EHR platforms. Second, in rheumatology, treatment goals are often defined using subjective outcome parameters, different from arterial hypertension and diabetes. This implies the necessary consideration of comorbidities, psychological and mental 
conditions possibly influencing the subjective assessment of disease activity.

\section{Role of nursing in rheumatologic care}

In several European countries, nursing has developed and specialized in the area of rheumatology (59-61). Specialized rheumatology nurses provide advice over the phone and offer self-management, education, and counseling for patients (6267). In addition, they participate in disease management, monitor disease-modifying treatments, help treat comorbidities and, after special training, give intra-articular injections and prescribe drug treatments $(59,68-71)$.

In 2012, the EULAR recommendations on the role of nurses in the treatment of inflammatory arthritis were first published (72). The 10 evidence-based recommendations provided a basis for an improved and standardized level of professional care across Europe. The most important recommendations relate to rheumatological care availability, patient training, including self-management, evidence-based disease monitoring and management, patient satisfaction, and psychosocial support. In addition, three recommendations focused on professional support for nurses: availability of guidelines or protocols, access to training to take on more advanced tasks. The assessment of these recommendations showed a high degree of agreement between the countries, but there were significant differences in their application, suggesting that the implementation was not comprehensive (72-74). The EULAR recommendations were then updated in 2018 and expanded to include three new overarching principles relevant to all eight recommendations (41). This update confirmed the contribution of rheumatology nurses to health care, including telemedicine, thus offering new opportunities for accessible and sustainable health care (67). Person-centered care and partnership with patients are considered essential dimensions that promote trust in care (75-79).

\section{Additional quality aspects like polypharmacy}

The T2T strategy does not comprehensively include all quality aspects of managing rheumatologic patients. Polypharmacy is a typical example of an additional clinical aspect to be managed in routine practice, with possible need of quality improvement. There are several definitions of polypharmacy (80), including the number of drugs (usually over 4) and their inappropriateness $(81,82)$. Due to the different definitions, the number of patients affected by polypharmacy varies considerably (81).

Polypharmacy can lead to adverse drug interactions (83) and increase severe adverse events, thereby increasing healthcare costs (84). In elderly patients over 65 years of age, $50 \%$ will be prescribed more than 6 drugs, and nearly 20\% will receive inappropriate medication (84). Comorbidities usually require additional treatment and may even lead to the prescription of unnecessary medication (85). So far, only a few RA studies have focused on polypharmacy in the literature: In a retrospective study on polypharmacy and comorbidities in Central European RA-patients, polypharmacy ( $\geq 5$ drugs) was found in $34 \%$ of patients, especially in female patients over 50 years of age (42). This percentage is lower than $44.4-67.9 \%$ of all RA patients as reported in previous literature (86-88). Possible reasons are differences in the insurance systems or the definition of polypharmacy $(82,89)$. The main limitation is the retrospective design with incomplete data sets and the relatively small sample size of 175 RA-patients.

New to this study was the use of the international Anatomical Therapeutic Chemical (ATC) codes to classify drugs (42). This coding has not previously been used for the polypharmacy approach in RA, although ATC coding supports differentiation between disease-specific treatment and treatment of comorbidities and the study of additional pharmacological aspects during follow-up.

\section{Data acquisition to provide quality indicators}

Standardization of data acquisition is crucial for implementing quality efforts and applying QIs in daily routine practice. Although guidelines for data collection and outcome measurements have been developed (34), such initiatives have not consistently been implemented in RA $(90,91)$, and rarely in other diseases.

The principle feasibility of standardized data acquisition in routine clinical care is shown in Denmark (DANBIO) (92). Also, the Swiss Clinical Quality Management in Rheumatic Diseases (SCQM) project collects long-term follow-up data of patients with several pre-defined rheumatic diseases. Compared to these prospective approaches, retrospective data-independent from the technique used-should be considered lower quality data (93). In prospective approaches, any core data set implemented in registries, research cohorts, or systems should ideally balance clinical feasibility and the potential to generate valuable research data. For this purpose, a Delphi consensus-based checklist was proposed to provide data both for registries and routine clinical care (94).

As an example, a study systematically evaluated the American College of Rheumatology's (ACR) QI measures for monitoring RA and therapy with methotrexate (MTX) (32). For this purpose, six ACR-QIs were analyzed using the EHRs from several locations. Three ACR QIs focused on the management, and three ACR QIs focused on the use of MTX. As a result, EHRs supported easy tracking of laboratory values, but most EHRs could not collect and process information from both patients and physicians. The authors, therefore, suggested the use of validated software tools to collect and analyze this information, preferably in an integrated system as in the SCQM project.

\section{DISCUSSION}

Because of the many efforts to improve health care quality, it is difficult to identify key issues for quality initiatives. Indeed, there is a wide field of quality efforts in rheumatology, with quite diverse challenges to put the most important quality efforts into clinical practice. This work applied new text analyses in current literature, finally providing 5 key elements as quality needs for future initiatives.

First, the statistical text analysis using the new Voyant Tools (https://voyant-tools.org/) of titles and abstracts showed that the 
number of selected publications was small but related to the topic of interest-with high Pearsson correlation coefficients.

According to the Collocate Graph analysis of the same software, it appears that so far, the WHO dimensions of physical, psychological and social aspects required for the concept of health, together with the educational aspect, are not routinely linked to quality initiatives in the literature. This lack of coding literature according to quality issues can be even expanded to the use of the Donabedian model in rheumatology. Consequently, it will be essential for future searches to fully respect these issues, thus providing the information needed to address these quality issues in routine clinical practice fully.

The 5 key elements for rheumatology quality filtered out from the literature have to be discussed more in detail:

(1) QIs for clinical routine: This overview article does not offer any detailed knowledge about the implementation of QS in rheumatology, but it is necessary to point out the need for corresponding QIs, with which quality improvements can then be measured. Both QSs and QIs have to be validated (47) and include the needs and possibilities of those affected with the diseases and follow a pre-defined consensus process (94). Most available QSs and QIs were primarily developed in very well-developed health systems but should be used in all countries.

Measuring QIs will help to close quality and supply gaps (32). Based on adequate QIs, the PDCA cycle then offers a practical approach to identifying and improving the quality of care $(2,95)$. For example, there is hardly any evidence available for some critical areas of quality improvement, such as the referral process. While the delay in diagnosis represents an essential gap in daily care, the challenge is that referral systems vary worldwide, and there are no indications of the feasible optimum of time (37). That is why the implementation and consecutive adaptions of QS in PDCA circles is crucial, and several components such as data sources, target group, and reporting period must be defined. The inclusion of different stakeholders (e.g., general practitioners, physiotherapists, patients, and pharmacists) can further increase the acceptance of such QS and QIs. Furthermore, focusing on the key areas of referral, disease assessment, therapy, education / self-management and comorbidities increases the likelihood of a significant improvement in the quality of care (37).

(2) Treat to target as treatment strategy: Despite the attractiveness of this strategy, it is not easy to translate it into routine clinical practice. In many cases, successful implementation requires structural quality with adequate human resources and process improvement with the definition of a suitable treatment goal with patient participation, regular monitoring of disease activity, and appropriate therapy adjustment (43). A good structural, personnel and technical infrastructure is required, including a software platform suitable for documentation and progress monitoring. The use of EHRs would support the implementation of the T2T strategy even better (96). A link with data on drug dispensing in pharmacies should also be sought in order to be able to better estimate the actual point in time of treatment changes. The results of audits and feedback could then be used to drive quality improvements (36).

(3) Role of nurses in rheumatologic care: Differences in the qualifications of rheumatology teams in different health systems determine the optimal use of the various professional groups and can also affect the quality of care provided to the patients (97). Indeed, the training and continuing education of nursing in rheumatology is not standardized in all European countries. Even the definition of "care" differs between countries. In the UK, for example, nursing has been defined as a practice model in which nurses, in collaboration with doctors and physiotherapists, occupational therapists and psychologists, provide education, monitoring and support for a specific group of patients (98). In any case, training should reflect the nurses' duties and activities (99).

Nowadays, it is planned to align the level of competence across Europe. Initiatives to implement the EULARrecommendations are essential and require national and international support from stakeholders such as the EULAR (41). Additional recommendations are needed that focus on other rheumatological diseases, including vasculitides. Together, these recommendations will further establish the importance of care in rheumatology and contribute to a standardized level of professional care across Europe.

(4) Additional quality aspects like polypharmacy: The application of drugs plays an essential role in managing rheumatic diseases. Polypharmacy is only one specific aspect, together with patients' compliance, number of comorbidities and monitoring of pharmacological side-effects during routine clinical management. Many of these aspects have not been well-researched and need further investigation (42).

(5) Data acquisition to provide QIs: For the future, EHRs should guide the relevant, diagnosis-specific QIs to be assessed quickly and easily in clinical practice $(32,51,96)$. Such extended use of EHRs offers the possibility to evaluate clinical courses together with patients' perceptions of their outcome and perceived health care. Especially process- and outcome-related QIs have to be validated as instruments to provide transparency and help to standardize quality assessment (96).

Indeed, health systems are increasingly using QIs directly extracted from EHRs. These measurements can then be used to evaluate standard care considering the outcome. Although electronic quality measures show promise in supporting to achieve potential goals of quality interventions, current studies suggest that significant infrastructure and analytical support at the practice level is necessary to integrate such electronic quality measurements into the existing health care systems (33). For rheumatic diseases with multiorgan manifestations and multiple disciplines, such standardized data acquisition should be carried out on an interdisciplinary platform.

At present, the user-oriented functionality of EHRs remains an obstacle, as cumbersome documentation, incorrect or repetitive data in drug lists and clinical notes, and lack of 
interoperability between systems of different physicians and health care providers can lead to reduced data quality. Expanding the collection of reliable data will result in improved reporting of rheumatologic quality (100-105), but requires user-oriented overall planning (96).

\section{Limitations}

Since this work did not seek additional involvement from external stakeholders such as administration and insurance, the overview of evidence-based quality efforts in rheumatology was only addressed from the medical perspective. In addition, screening of literature by an independent expert would increase the objectivity of selected publications. Also, in the content analysis, strategies and application goals were summarized without details for their implementation.

Another limitation is that few studies were identified. This can be explained by the selection of the inclusion and exclusion criteria. Also, publications do not necessarily list possible links to quality aspects and refer to general medical problems more than to specific rheumatologic issues unless the work specifically addresses a certain rheumatologic disease. This aspect was taken into account by additional manual selection of important publications.

As a financial limitation of the overview, it should be mentioned that not freely available publications which were classified as being of little relevance due to their abstract were excluded from further review $(n=59)$. Thus, a limitation of the text analysis is that not all of the preselected texts were available in full-text format and therefore could not be analyzed. The generally recognized publication bias should be cited as a limitation, as it is more likely that statistically significant results will be published than studies that contain insignificant results or outline errors.

\section{Outlook}

As conventional, unstructured routine documentation isdespite the possible use of automatic text search optionsinsufficient to address most rheumatologic quality efforts in clinical practice, future projects are required to standardize the documentation of all disease-specific QIs. It can be anticipated that using these QIs for direct patient care data acquisition will allow various future quality projects.

Although many approaches to quality improvement have been studied, the proposed interventions were subsequently not or only partially implemented in practice for reasons of practicality. This question, however, is of essential importance for further development of quality assurance in rheumatology and should always be addressed in parallel with the development of new quality approaches. While clinical studies usually follow an "isolated" approach with only one intervention for possible quality improvement over a manageable time frame, clinical settings vary in routine practice, and multidimensional approaches with long-term data are needed.
Quality efforts are currently carried out primarily in the in-patient area. It is essential for rheumatologic patients to comprehensively prepare and implement such quality efforts in an interdisciplinary manner and for the outpatient area. Aspects of the structure, procedural and outcome quality must be considered for in-patient settings.

Based on the findings above, future quality projects are needed, especially in the following fields of rheumatologic interest:

1. Initiatives to define the treat to target goals in all rheumatologic diseases:

Definitions of T2T goals are necessary for all rheumatic diseases. Definition of disease-specific quality standards is decisive for implementing future quality efforts. It will be helpful to involve nursing staff, other medical disciplines and patients early in the developmental process, to enable implementation of quality efforts in the long term.

The T2T goals can then be expanded to fulfill the health goals defined by the WHO.

2. Support of secondary quality goals such as avoidance of polypharmacy.

At present, quality goals such as avoiding polypharmacy are challenging to achieve in clinical practice. However, since drug application plays an essential role in treating rheumatic diseases and comorbidities, both the number of possible sideeffects and drug interactions can be examined to simplify patients' information. The use of ATC coding can certainly support such initiatives.

Other "secondary" quality efforts will reduce the number of untreated comorbidities or lower the number of necessary accompanying operations.

3. Comparison of quality dimensions between different care settings (outpatient alone or with in-patient support) for different rheumatic diseases.

Discussion about achievements of important quality goals will have to be conducted again and again. To acquire substantial evidence, EHR-based, rapidly updated, and disease-specific real-life data are needed to argue for quality-oriented strategic decisions. Thus, national and international comparisons between different institutions can contribute to the discussion of quality goals and assessments. Also, it will be possible to generate comparative data for individual disease management strategies in the future, thus contributing to the decision-making process for changes in therapy.

We foresee that the advantages and disadvantages of different care settings will be decided based on evidence in rheumatology. The value of long-term outcome parameters will then gain more and more essential and can be argued with real-life data.

In the long-term, the strategy of future research projects will have to address all health aspects defined by the WHO (physical, mental and social health). Such comprehensive approaches may then support both the social and the mental health sector. Health organizations will often be asked about such comprehensive 
models of health care that meet the quality goals for the patients, defined by evidence from research studies.

\section{AUTHOR CONTRIBUTIONS}

JP and MS contributed to the design, performed the work, analyzed the results, wrote, and finalized the manuscript.

\section{REFERENCES}

1. IOM. Crossing the Quality Chasm: A New Health System for the 21st Century. Washington, DC: IOM (2001).

2. Chow SL, Shojania KG. "Rheum to improve": quality improvement in outpatient rheumatology. J Rheumatol. (2017) 44:130410. doi: 10.3899 /jrheum. 161053

3. Suzuki Y, Uehara R, Tajima C, Noguchi A, Ide M, Ichikawa Y, et al. Elevation of serum hepatic aminotransferases during treatment of rheumatoid arthritis with low-dose methotrexate. Risk factors and response to folic acid. Scand J Rheumatol. (1999) 28:273-81. doi: 10.1080/03009749950155454

4. Edwards FD, Grover ML, Cook CB, Chang YH. Use of FRAX as a determinant for risk-based osteoporosis screening may decrease unnecessary testing while improving the odds of identifying treatment candidates. Womens Health Issues. (2014) 24:629-34. doi: 10.1016/j.whi.2014.06.006

5. Voshaar MJ, Nota I, van de Laar MA, van den Bemt BJ. Patient-centred care in established rheumatoid arthritis. Best Pract Res Clin Rheumatol. (2015) 29:643-63. doi: 10.1016/j.berh.2015.09.007

6. Anderson E, Bajaj P, Raghavan S, Patnaik A, Roppelt H. Assessment of American college of rheumatology-endorsed quality indicators in rheumatoid arthritis patients: a quality improvement initiative. J Clin Rheumatol. (2016) 22:63-7. doi: 10.1097/RHU.0000000000000323

7. Dorner T, Strand V, Cornes P, Goncalves J, Gulacsi L, Kay J, et al. The changing landscape of biosimilars in rheumatology. Ann Rheum Dis. (2016) 75:974-82. doi: 10.1136/annrheumdis-2016-209166

8. Gulacsi L, Rencz F, Poor G, Szekanecz Z, Brodszky V, Baji P, et al. Patients' access to biological therapy in chronic inflammatory conditions; per capita GDP does not explain the intercountry differences. Ann Rheum Dis. (2016) 75:942-3. doi: 10.1136/annrheumdis-2015-208741

9. Hazlewood GS, Barr SG, Lopatina E, Marshall DA, Lupton TL, Fritzler $\mathrm{MJ}$, et al. Improving appropriate access to care with central referral and triage in rheumatology. Arthritis Care Res (Hoboken). (2016) 68:154753. doi: 10.1002/acr.22845

10. Barnabe C, Jones CA, Bernatsky S, Peschken CA, Voaklander D, Homik J, et al. Inflammatory arthritis prevalence and health services use in the first nations and non-first nations populations of Alberta, Canada. Arthritis Care Res (Hoboken). (2017) 69:467-74. doi: 10.1002/acr.22959

11. Lesuis N, den Broeder AA, van Vollenhoven RF, Vriezekolk JE, Hulscher M. Choosing wisely in daily practice: a mixed methods study on determinants of antinuclear antibody testing by rheumatologists. Scand J Rheumatol. (2017) 46:241-6. doi: 10.1080/03009742.2016.1190983

12. Grimshaw JM, Thomas RE, MacLennan G, Fraser C, Ramsay CR, Vale L, et al. Effectiveness and efficiency of guideline dissemination and implementation strategies. Health Technol Assess. (2004) 8:iii-iv, 172. doi: $10.3310 /$ hta 8060

13. Porter ME. What is value in health care? N Engl J Med. (2010) 363:247781. doi: 10.1056/NEJMp1011024

14. Brilli RJ, Allen S, Davis JT. Revisiting the quality chasm. Pediatrics. (2014) 133:763-5. doi: 10.1542/peds.2013-3090

15. Patwardhan M, Fisher DA, Mantyh CR, McCrory DC, Morse MA, Prosnitz RG, et al. Assessing the quality of colorectal cancer care: do we have appropriate quality measures? (A systematic review of literature). J Eval Clin Pract. (2007) 13:831-45. doi: 10.1111/j.1365-2753.2006.00762.x

16. Gooiker GA, Kolfschoten NE, Bastiaannet E, van de Velde CJ, Eddes EH, van der Harst E, et al. Evaluating the validity of quality indicators for colorectal cancer care. J Surg Oncol. (2013) 108:465-71. doi: 10.1002/jso.23420
All authors contributed to the article and approved the submitted version.

\section{FUNDING}

Publication fee was funded by the Medical University of Innsbruck.

17. Elliot C, McUllagh C, Brydon M, Zwi K. Developing key performance indicators for a tertiary children's hospital network. Aust Health Rev. (2018) 42:491-500. doi: 10.1071/AH17263

18. Gilbert SM. Revisiting structure, process, and outcome. Cancer. (2015) 121:328-30. doi: 10.1002/cncr.29070

19. Saag KG, Olivieri JJ, Patino F, Mikuls TR, Allison JJ, MacLean CH. Measuring quality in arthritis care: the Arthritis Foundation's quality indicator set for analgesics. Arthritis Rheum. (2004) 51:337-49. doi: 10.1002/art.20422

20. Barber CE, Marshall DA, Mosher DP, Akhavan P, Tucker L, Houghton K, et al. Development of system-level performance measures for evaluation of models of care for inflammatory arthritis in Canada. J Rheumatol. (2016) 43:530-40. doi: 10.3899/jrheum.150839

21. Lovell DJ, Passo MH, Beukelman T, Bowyer SL, Gottlieb BS, Henrickson M, et al. Measuring process of arthritis care: a proposed set of quality measures for the process of care in juvenile idiopathic arthritis. Arthritis Care Res (Hoboken). (2011) 63:10-6. doi: 10.1002/acr.20348

22. Harris JG, Bingham CA, Morgan EM. Improving care delivery and outcomes in pediatric rheumatic diseases. Curr Opin Rheumatol. (2016) 28:1106. doi: 10.1097/BOR.0000000000000257

23. Yazdany J, Myslinski R, Miller A, Francisco M, Desai S, Schmajuk G, et al. Methods for developing the American college of rheumatology's electronic clinical quality measures. Arthritis Care Res (Hoboken). (2016) 68:14029. doi: 10.1002/acr.22985

24. Yazdany J, Robbins M, Schmajuk G, Desai S, Lacaille D, Neogi T, et al. Development of the American college of rheumatology's rheumatoid arthritis electronic clinical quality measures. Arthritis Care Res (Hoboken). (2016) 68:1579-90. doi: 10.1002/acr.22984

25. Yazdany J, Bansback N, Clowse M, Collier D, Law K, Liao KP, et al. Rheumatology informatics system for effectiveness: a national informaticsenabled registry for quality improvement. Arthritis Care Res (Hoboken). (2016) 68:1866-73. doi: 10.1002/acr.23089

26. Ouzzani M, Hammady H, Fedorowicz Z, Elmagarmid A. Rayyana web and mobile app for systematic reviews. Syst Rev. (2016) 5:210. doi: 10.1186/s13643-016-0384-4

27. Moher D, Booth A, Stewart L. How to reduce unnecessary duplication: use PROSPERO. BJOG. (2014) 121:784-6. doi: 10.1111/1471-0528.12657

28. De Caro W, Mitello L, Marucci AR, Lancia L, Sansoni J. Textual analysis and data mining: an interpreting research on nursing. Stud Health Technol Inform. (2016) 225:948. doi: 10.3233/978-1-61499-658-3-948

29. Hetenyi G, Lengyel A, Szilasi M. Quantitative analysis of qualitative data: using voyant tools to investigate the sales-marketing interface. J Indust Eng Manag. (2019) 12:393. doi: 10.3926/jiem.2929

30. Page MJ, McKenzie JE, Bossuyt PM, Boutron I, Hoffmann TC, Mulrow CD, et al. The PRISMA 2020 statement: an updated guideline for reporting systematic reviews. BMJ. (2021) 372:n71. doi: 10.1016/j.jclinepi.2021.03.001

31. van Hulst LT, Fransen J, den Broeder AA, Grol R, van Riel PL, Hulscher ME. Development of quality indicators for monitoring of the disease course in rheumatoid arthritis. Ann Rheum Dis. (2009) 68:180510. doi: 10.1136/ard.2009.108555

32. Adhikesavan LG, Newman ED, Diehl MP, Wood GC, Bili A. American College of Rheumatology quality indicators for rheumatoid arthritis: benchmarking, variability, and opportunities to improve quality of care using the electronic health record. Arthritis Rheum. (2008) 59:170512. doi: 10.1002 /art. 24054

33. FitzGerald JD, Mikuls TR, Neogi T, Singh JA, Robbins M, Khanna PP, et al. Development of the American College of Rheumatology electronic 
clinical quality measures for gout. Arthritis Care Res (Hoboken). (2018) 70:659-71. doi: 10.1002/acr.23500

34. Radner H, Chatzidionysiou K, Nikiphorou E, Gossec L, Hyrich KL, Zabalan C, et al. 2017 EULAR recommendations for a core data set to support observational research and clinical care in rheumatoid arthritis. Ann Rheum Dis. (2018) 77:476-9. doi: 10.1136/annrheumdis-2017-212256

35. Liu LH, Choden S, Yazdany J. Quality improvement initiatives in rheumatology: an integrative review of the last 5 years. Curr Opin Rheumatol. (2019) 31:98-108. doi: 10.1097/BOR.0000000000000586

36. Barber CEH, Mosher D, Dowling S, Bohm V, Solbak NM, MacMullan $\mathrm{P}$, et al. Implementation and evaluation of audit and feedback for monitoring treat-to-target (T2T) strategies in rheumatoid arthritis using performance measures. Rheumatol Ther. (2020) 7:909-25. doi: 10.1007/s40744-020-00237-0

37. Kiltz U, Landewe RBM, van der Heijde D, Rudwaleit M, Weisman MH, Akkoc N, et al. Development of ASAS quality standards to improve the quality of health and care services for patients with axial spondyloarthritis. Ann Rheum Dis. (2020) 79:193-201. doi: 10.1136/annrheumdis-2019-216034

38. Patel K, Chim YL, Grant J, Wascher M, Nathanson A, Canfield S. Development and implementation of clinical outcome measures for automated collection within specialty pharmacy practice. J Manag Care Spec Pharm. (2020) 26:901-9. doi: 10.18553/jmcp.2020.26.7.901

39. Doubova SV, Perez-Cuevas R. Quality of care for hip and knee osteoarthritis at family medicine clinics: lessons from Mexico. Int J Qual Health Care. (2015) 27:125-31. doi: 10.1093/intqhe/mzv003

40. Donnelly S, Manning M, Mannan H, Wilson AG, Kroll T. Renegotiating dimensions of the self: a systematic review and qualitative evidence synthesis of the lived experience of self-managing rheumatoid arthritis. Health Expect. (2020) 23:1388-411. doi: 10.1111/hex.13122

41. Bech B, Primdahl J, van Tubergen A, Voshaar M, Zangi HA, Barbosa L, et al. 2018 update of the EULAR recommendations for the role of the nurse in the management of chronic inflammatory arthritis. Ann Rheum Dis. (2020) 79:61-8. doi: 10.1136/annrheumdis-2019-215458

42. Jack JD, McCutchan R, Maier S, Schirmer M. Polypharmacy in middleEuropean rheumatoid arthritis-patients: a retrospective longitudinal cohort analysis with systematic literature review. Front Med (Lausanne). (2020) 7:573542. doi: $10.3389 /$ fmed.2020.573542

43. Solomon DH, Lee SB, Zak A, Corrigan C, Agosti J, Bitton A, et al. Implementation of treat-to-target in rheumatoid arthritis through a learning collaborative: rationale and design of the TRACTION trial. Semin Arthritis Rheum. (2016) 46:81-7. doi: 10.1016/j.semarthrit.2016.02.009

44. Ferlie EB, Shortell SM. Improving the quality of health care in the United Kingdom and the United States: a framework for change. Milbank Q. (2001) 79:281-315. doi: 10.1111/1468-0009.00206

45. Guidelines I. o.M.U.C.t.A.t.P.H.S.o.C.P. Clinical Practice Guidelines: Directions for a New Program, eds. Field MJ, Lohr KN. Washington, DC: Guidelines I. o.M.U.C.t.A.t.P.H.S.o.C.P (1990).

46. Woolf SH, Grol R, Hutchinson A, Eccles M, Grimshaw J. Clinical guidelines: potential benefits, limitations, and harms of clinical guidelines. BMJ. (1999) 318:527-30. doi: 10.1136/bmj.318.7182.527

47. Wilson BA, Cooper M, Barber CEH. Standards of care for inflammatory arthritis: a literature review. Semin Arthritis Rheum. (2017) 47:228. doi: 10.1016/j.semarthrit.2017.02.009

48. Desai SP, Yazdany J. Quality measurement and improvement in rheumatology: rheumatoid arthritis as a case study. Arthritis Rheum. (2011) 63:3649-60. doi: 10.1002/art.30605

49. Mata-Cases M, Roura-Olmeda P, Berengue-Iglesias M, BirulesPons M, Mundet-Tuduri X, Franch-Nadal J, et al. Fifteen years of continuous improvement of quality care of type 2 diabetes mellitus in primary care in Catalonia, Spain. Int J Clin Pract. (2012) 66:289-98. doi: 10.1111/j.1742-1241.2011.02872.x

50. Ryan AM, Doran T. The effect of improving processes of care on patient outcomes: evidence from the United Kingdom's quality and outcomes framework. Med Care. (2012) 50:1919. doi: 10.1097/MLR.0b013e318244e6b5

51. Navarro-Compan V, Smolen JS, Huizinga TW, Landewe R, Ferraccioli G, da Silva JA, et al. Quality indicators in rheumatoid arthritis: results from the METEOR database. Rheumatology (Oxford). (2015) 54:16309. doi: 10.1093/rheumatology/kev108

52. Marshall MN, Shekelle PG, McGlynn EA, Campbell S, Brook RH, Roland MO. Can health care quality indicators be transferred between countries? Qual Saf Health Care. (2003) 12:8-12. doi: 10.1136/qhc.12.1.8

53. Smolen JS, Aletaha D, Bijlsma JW, Breedveld FC, Boumpas D, Burmester G, et al. Treating rheumatoid arthritis to target: recommendations of an international task force. Ann Rheum Dis. (2010) 69:631-7. doi: 10.1136/ard.2009.123919

54. Bombardier C, Hazlewood GS, Akhavan P, Schieir O, Dooley A, Haraoui $\mathrm{B}$, et al. Canadian Rheumatology Association recommendations for the pharmacological management of rheumatoid arthritis with traditional and biologic disease-modifying antirheumatic drugs: part II safety. J Rheumatol. (2012) 39:1583-602. doi: 10.3899/jrheum.120165

55. Singh JA, Saag KG, Bridges SL, Jr., Akl EA, Bannuru RR, Sullivan MC, et al. 2015 American college of rheumatology guideline for the treatment of rheumatoid arthritis. Arthritis Rheumatol. (2016) 68:126. doi: 10.1002/art.39480

56. Smolen JS, Landewe RBM, Bijlsma JWJ, Burmester GR, Dougados M, Kerschbaumer A, et al. EULAR recommendations for the management of rheumatoid arthritis with synthetic and biological disease-modifying antirheumatic drugs: 2019 update. Ann Rheum Dis. (2020) 79:68599. doi: 10.1136/annrheumdis-2019-216655

57. Haraoui B, Bensen W, Bessette L, Le Clercq S, Thorne C, Wade J. Treating rheumatoid arthritis to target: a Canadian physician survey. J Rheumatol. (2012) 39:949-53. doi: 10.3899/jrheum.111134

58. Ivers N, Jamtvedt G, Flottorp S, Young JM, Odgaard-Jensen J, French SD, et al. Audit and feedback: effects on professional practice and healthcare outcomes. Cochrane Database Syst Rev. (2012) 6:CD000259. doi: 10.1002/14651858.CD000259.pub3

59. Meadows A, Sheehan NJ. Prescribing and injecting: the expanding role of the rheumatology nurse. Musculoskeletal Care. (2005) 3:1768. doi: $10.1002 / \mathrm{msc} .7$

60. Stamm T, Hill J. Extended roles of non-physician health professionals and innovative models of care within Europe: results from a web-based survey. Musculoskeletal Care. (2011) 9:93-101. doi: 10.1002/msc.201

61. de Thurah A, Esbensen BA, Roelsgaard IK, Frandsen TF, Primdahl J. Efficacy of embedded nurse-led versus conventional physician-led follow-up in rheumatoid arthritis: a systematic review and meta-analysis. RMD Open. (2017) 3:e000481. doi: 10.1136/rmdopen-2017-000481

62. Thwaites C, Ryan S, Hassell A. A survey of rheumatology nurse specialists providing telephone helpline advice within England and Wales. Rheumatology (Oxford). (2008) 47:5225. doi: 10.1093/rheumatology/ken041

63. Gronning K, Rannestad T, Skomsvoll JF, Rygg LO, Steinsbekk A. Long-term effects of a nurse-led group and individual patient education programme for patients with chronic inflammatory polyarthritis - a randomised controlled trial. J Clin Nurs. (2014) 23:1005-17. doi: 10.1111/jocn.12353

64. Primdahl J, Sorensen J, Horn HC, Petersen R, Horslev-Petersen K. Shared care or nursing consultations as an alternative to rheumatologist followup for rheumatoid arthritis outpatients with low disease activity-patient outcomes from a 2-year, randomised controlled trial. Ann Rheum Dis. (2014) 73:357-64. doi: 10.1136/annrheumdis-2012-202695

65. Dougados M, Soubrier M, Perrodeau E, Gossec L, Fayet F, Gilson M, et al. Impact of a nurse-led programme on comorbidity management and impact of a patient self-assessment of disease activity on the management of rheumatoid arthritis: results of a prospective, multicentre, randomised, controlled trial (COMEDRA). Ann Rheum Dis. (2015) 74:172533. doi: 10.1136/annrheumdis-2013-204733

66. Zangi HA, Ndosi M, Adams J, Andersen L, Bode C, Bostrom $\mathrm{C}$, et al. EULAR recommendations for patient education for people with inflammatory arthritis. Ann Rheum Dis. (2015) 74:954-62. doi: 10.1136/annrheumdis-2014-206807

67. de Thurah A, Stengaard-Pedersen K, Axelsen M, Fredberg U, Schougaard LMV, Hjollund NHI, et al. Tele-health followup strategy for tight control of disease activity in rheumatoid arthritis: results of a randomized controlled trial. Arthritis Care Res (Hoboken). (2018) 70:353-60. doi: 10.1002/acr.23280 
68. Palmer D, El Miedany Y. Biological nurse specialist: goodwill to good practice. Br J Nurs. (2010) 19:477-80. doi: 10.12968/bjon.2010.19.8.47632

69. Primdahl J, Clausen J, Horslev-Petersen K. Results from systematic screening for cardiovascular risk in outpatients with rheumatoid arthritis in accordance with the EULAR recommendations. Ann Rheum Dis. (2013) 72:1771-6. doi: 10.1136/annrheumdis-2013-203682

70. Primdahl J, Ferreira RJ, Garcia-Diaz S, Ndosi M, Palmer D, van Eijk-Hustings Y. Nurses' role in cardiovascular risk assessment and management in people with inflammatory arthritis: a European perspective. Musculoskeletal Care. (2016) 14:133-51. doi: 10.1002/msc.1121

71. Lion V, Schirmer M. Nurses' roles in the management of chronic inflammatory arthritis: a systematic review. Rheumatol Int. (2018) 38:202736. doi: 10.1007/s00296-018-4135-9

72. van Eijk-Hustings Y, van Tubergen A, Bostrom C, Braychenko E, Buss B, Felix J, et al. EULAR recommendations for the role of the nurse in the management of chronic inflammatory arthritis. Ann Rheum Dis. (2012) 71:13-9. doi: 10.1136/annrheumdis-2011-200185

73. Barbosa L, Ramiro S, Santos MJ, Canas Da Silva J. Applicability of the EULAR recommendations on the role of the nurse in the management of chronic inflammatory arthritis in Portugal. Acta Reumatol Port. (2013) 38:186-91.

74. Fusama M, Nakahara H, van Eijk-Hustings Y, Oliver S, Takeuchi T. Survey on attitudes regarding EULAR recommendations for the role of nurses involved in medical care of patients with chronic inflammatory arthritis in Japan. Mod Rheumatol. (2017) 27:886-93. doi: 10.1080/14397595.2016.1260199

75. Primdahl J, Wagner L, Horslev-Petersen K. Being an outpatient with rheumatoid arthritis-a focus group study on patients' selfefficacy and experiences from participation in a short course and one of three different outpatient settings. Scand J Caring Sci. (2011) 25:394-403. doi: 10.1111/j.1471-6712.2010.00854.x

76. Bala SV, Samuelson K, Hagell P, Svensson B, Fridlund B, Hesselgard K. The experience of care at nurse-led rheumatology clinics. Musculoskeletal Care. (2012) 10:202-11. doi: 10.1002/msc.1021

77. Larsson I, Bergman S, Fridlund B, Arvidsson B. Patients' experiences of a nurse-led rheumatology clinic in Sweden: a qualitative study. Nurs Health Sci. (2012) 14:501-7. doi: 10.1111/j.1442-2018.2012.00723.x

78. Vinall-Collier K, Madill A, Firth J. A multi-centre study of interactional style in nurse specialist- and physician-led Rheumatology clinics in the UK. Int $J$ Nurs Stud. (2016) 59:41-50. doi: 10.1016/j.ijnurstu.2016.02.009

79. Larsson I. Nurse-led care and patients as partners are essential aspects of the future of rheumatology care. J Rheumatol. (2017) 44:7202. doi: $10.3899 /$ jrheum. 170215

80. Hajjar ER, Cafiero AC, Hanlon JT. Polypharmacy in elderly patients. Am J Geriatr Pharmacother. (2007) 5:34551. doi: 10.1016/j.amjopharm.2007.12.002

81. Bushardt RL, Massey EB, Simpson TW, Ariail JC, Simpson KN. Polypharmacy: misleading, but manageable. Clin Interv Aging. (2008) 3:3839. doi: $10.2147 /$ CIA.S2468

82. Masnoon N, Shakib S, Kalisch-Ellett L, Caughey GE. What is polypharmacy? A systematic review of definitions. BMC Geriatr. (2017) 17:230. doi: 10.1186/s12877-017-0621-2

83. Pflugbeil S, Bockl K, Pongratz R, Leitner M, Graninger W, Ortner A. Drug interactions in the treatment of rheumatoid arthritis and psoriatic arthritis. Rheumatol Int. (2020) 40:511-21. doi: 10.1007/s00296-020-04526-3

84. Fialova D, Topinkova E, Gambassi G, Finne-Soveri H, Jonsson PV, Carpenter I, et al. Potentially inappropriate medication use among elderly home care patients in Europe. JAMA. (2005) 293:1348-58. doi: 10.1001/jama.293.11.1348

85. Kronzer VL, Crowson CS, Sparks JA, Myasoedova E, Davis JM, III. Comorbidities as risk factors for rheumatoid arthritis and their accrual after diagnosis. Mayo Clin Proc. (2019) 94:2488-98. doi: 10.1016/j.mayocp.2019.08.010

86. Gonzalez-Gamboa LM, Barocio-Ramirez AK, Rocha-Munoz AD, de SantosAvila F, Meda-Lara RM, Gonzalez-Lopez L, et al. Disease activity score on 28 joints and polypharmacy are independent predictors for health-related quality of life evaluated by INCAVISA in patients with rheumatoid arthritis. J Clin Rheumatol. (2016) 22:399-404. doi: 10.1097/RHU.00000000000 00463
87. Gomides APM, Albuquerque CP, Santos ABV, Amorim RBC, Bertolo MB, Junior PL, et al. High levels of polypharmacy in rheumatoid arthritis-A challenge not covered by current management recommendations: data from a large real-life study. J Pharm Pract. (2019) 34:897190019869158. doi: 10.1177/0897190019869158

88. Ma SN, Zaman Huri H, Yahya F. Drug-related problems in patients with rheumatoid arthritis. Ther Clin Risk Manag. (2019) 15:50524. doi: 10.2147/TCRM.S194921

89. Treharne GJ, Douglas KM, Iwaszko J, Panoulas VF, Hale ED, Mitton DL, et al. Polypharmacy among people with rheumatoid arthritis: the role of age, disease duration and comorbidity. Musculoskeletal Care. (2007) 5:17590. doi: $10.1002 / \mathrm{msc} .112$

90. Aletaha D, Landewe R, Karonitsch T, Bathon J, Boers M, Bombardier C, et al. Reporting disease activity in clinical trials of patients with rheumatoid arthritis: EULAR/ACR collaborative recommendations. Ann Rheum Dis. (2008) 67:1360-4. doi: 10.1136/ard.2008.091454

91. Buch MH, Silva-Fernandez L, Carmona L, Aletaha D, Christensen R, Combe B, et al. Development of EULAR recommendations for the reporting of clinical trial extension studies in rheumatology. Ann Rheum Dis. (2015) 74:963-9. doi: 10.1136/annrheumdis-2013-204948

92. Hetland ML. DANBIO-powerful research database and electronic patient record. Rheumatology (Oxford). (2011) 50:69-77. doi: 10.1093/rheumatology/keq309

93. Stekhoven D, Scherer A, Nissen MJ, Grobety V, Yawalkar N, Villiger PM, et al. Hypothesis-free analyses from a large psoriatic arthritis cohort support merger to consolidated peripheral arthritis definition without subtyping. Clin Rheumatol. (2017) 36:2035-43. doi: 10.1007/s10067-017-3637-2

94. Veraar C, Hasler P, Schirmer M. A multidisciplinary Delphi consensusbased checklist to define clinical documentation tools for both routine and research purposes. Health Serv Res Manag Epidemiol. (2018) 5:2333392817754161. doi: 10.1177/2333392817754161

95. Reed JE, Card AJ. The problem with plan-do-study-act cycles. BMJ Qual Saf. (2016) 25:147-52. doi: 10.1136/bmjqs-2015-005076

96. Pallua J, Schirmer M. Rolle der Dokumentations-IT in der Rheumatologie. Rheuma Plus. (2021) 4:179-84. doi: 10.1007/s12688-021-00396-0

97. Buchan J, Dal Poz MR. Skill mix in the health care workforce: reviewing the evidence. Bull World Health Organ. (2002) 80:575-80. doi: 10.1590/S0042-96862002000700010

98. Ndosi M, Vinall K, Hale C, Bird H, Hill J. The effectiveness of nurse-led care in people with rheumatoid arthritis: a systematic review. Int J Nurs Stud. (2011) 48:642-54. doi: 10.1016/j.ijnurstu.2011.02.007

99. Craig P, Dieppe P, Macintyre S, Michie S, Nazareth I, Petticrew M. Developing and evaluating complex interventions: the new Medical Research Council guidance. Int J Nurs Stud. (2013) 50:587-92. doi: 10.1016/j.ijnurstu.2012.09.010

100. Garvin JH, DuVall SL, South BR, Bray BE, Bolton D, Heavirland $\mathrm{J}$, et al. Automated extraction of ejection fraction for quality measurement using regular expressions in Unstructured Information Management Architecture (UIMA) for heart failure. J Am Med Inform Assoc. (2012) 19:859-66. doi: 10.1136/amiajnl-2011000535

101. Collinsworth AW, Masica AL, Priest EL, Berryman CD, Kouznetsova $\mathrm{M}$, Glorioso $\mathrm{O}$, et al. Modifying the electronic health record to facilitate the implementation and evaluation of a bundled care program for intensive care unit delirium. EGEMS (Wash DC). (2014) 2:1121. doi: 10.13063/2327-9214.1121

102. Gawron AJ, Thompson WK, Keswani RN, Rasmussen LV, Kho AN. Anatomic and advanced adenoma detection rates as quality metrics determined via natural language processing. Am J Gastroenterol. (2014) 109:1844-9. doi: 10.1038/ajg.2014.147

103. Newman ED, Lerch V, Billet J, Berger A, Kirchner HL. Improving the quality of care of patients with rheumatic disease using patient-centric electronic redesign software. Arthritis Care Res (Hoboken). (2015) 67:54653. doi: 10.1002/acr.22479

104. Raju GS, Lum PJ, Slack RS, Thirumurthi S, Lynch PM, Miller E, et al. Natural language processing as an alternative to manual reporting of colonoscopy quality metrics. Gastrointest Endosc. (2015) 82:5129. doi: 10.1016/j.gie.2015.01.049 
105. Tonner C, Schmajuk G, Yazdany J. A new era of quality measurement in rheumatology: electronic clinical quality measures and national registries. Curr Opin Rheumatol. (2017) 29:131-7. doi: 10.1097/BOR.0000000000000364

Conflict of Interest: The authors declare that the research was conducted in the absence of any commercial or financial relationships that could be construed as a potential conflict of interest.

Publisher's Note: All claims expressed in this article are solely those of the authors and do not necessarily represent those of their affiliated organizations, or those of the publisher, the editors and the reviewers. Any product that may be evaluated in this article, or claim that may be made by its manufacturer, is not guaranteed or endorsed by the publisher.

Copyright $\odot 2021$ Pallua and Schirmer. This is an open-access article distributed under the terms of the Creative Commons Attribution License (CC BY). The use, distribution or reproduction in other forums is permitted, provided the original author(s) and the copyright owner(s) are credited and that the original publication in this journal is cited, in accordance with accepted academic practice. No use, distribution or reproduction is permitted which does not comply with these terms. 